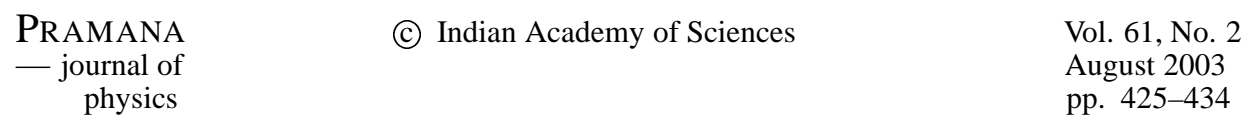

\title{
Segmented conjugated polymers
}

\author{
G PADMANABAN and S RAMAKRISHNAN \\ Department of Inorganic and Physical Chemistry, Indian Institute of Science, Bangalore 560012 , \\ India
}

\begin{abstract}
Segmented conjugated polymers, wherein the conjugation is randomly truncated by varying lengths of non-conjugated segments, form an interesting class of polymers as they not only represent systems of varying stiffness, but also ones where the backbone can be construed as being made up of chromophores of varying excitation energies. The latter feature, especially when the chromophores are fluorescent, like in MEHPPV, makes these systems particularly interesting from the photophysics point of view. Segmented MEHPPV- $x$ samples, where $x$ represents the mole fraction of conjugated segments, were prepared by a novel approach that utilizes a suitable precursor wherein selective elimination of one of the two eliminatable groups is affected; the uneliminated units serve as conjugation truncations. Control of the composition $x$ of the precursor therefore permits one to prepare segmented MEHPPV- $x$ samples with varying levels of conjugation (elimination). Using fluorescence spectroscopy, we have seen that even in single isolated polymer chains, energy migration from the shorter (higher energy) chromophores to longer (lower energy) ones occurs - the extent of which depends on the level of conjugation. Further, by varying the solvent composition, it is seen that the extent of energy transfer and the formation of poorly emissive inter-chromophore excitons are greatly enhanced with increasing amounts of non-solvent. A typical S-shaped curve represents the variation of emission yields as a function of composition suggestive of a cooperative collapse of the polymer coil, reminiscent of conformational transitions seen in biological macromolecules.
\end{abstract}

Keywords. Polyphenylene vinylene; segmented conjugated polymer; control of conjugation length; fluorescence; energy transfer; conformational transition.

PACS Nos 78.66.Qn; 81.16.Be; 82.35.Cd; 82.35.Jk

\section{Introduction}

Polyphenylene vinylene (PPV) and its derivatives have attracted a great deal of attention because of their fascinating photophysical properties such as light-emission [1,2], photoconductivity [3], lasing [4], photovoltaic effect [5], etc. The discovery that both their photoluminescence (PL) and electroluminescence (EL) spectra are essentially identical suggests that the state from which emission occurs is the same in both cases [6]. Hence, a great deal of attention has been devoted to the understanding of the fundamental photophysical process underlying the emission. Segmented conjugated polymers, where conjugated segments of varying lengths are separated electronically by insulating spacers, is one approach to control both the color and possibly also the efficiency of emission. Several earlier attempts to control conjugation lengths using time [7] and temperature of elimination, [8] and selective elimination in copolymer systems $[9,10]$ resulted only in limited success. A 
few years ago, we reported a novel approach for the statistical control of molecular conjugation length over a very wide range by a selective elimination of a suitable precursor containing two types of eliminatable groups [11]. Selective elimination of one of the substituents (such as acetate, xanthate) from the precursor polymer leaving the other (methoxy group) unaffected lead to the generation of segmented conjugated polymers. In this contribution, we review our efforts in the area of soluble segmented MEHPPV- $x$ [12,13] with particular emphasis on their solution photophysical properties specifically with regards to intra-chain energy transfer and solvent-induced conformational collapse.

\section{Experimental section}

The synthesis and characterization of the various segmented MEHPPV- $x$ samples via both the acetate [12] and xanthate [13] routes have been described earlier. The UV-visible and fluorescence spectra were recorded on a Hitachi U3400 and a Perkin Elmer LS-50B instrument, respectively. For the solvent titration studies, a calculated amount of a standard solution was diluted using appropriate amounts of the two selected solvents to ensure that the final concentration of the polymer in all the measurements was kept constant.

\section{Results and discussion}

\subsection{Synthesis and structural characterization}

The sulfonium chloride polyelectrolyte precursor of MEHPPV was prepared according to a slightly modified Wessling procedure. As described earlier, the polyelectrolyte was treated with varying mole ratios of the two nucleophiles, namely acetate (or xanthate) and methanol to give the required neutral precursor polymer (Scheme 1). The composition of the precursor copolymers, which was determined using ${ }^{1} \mathrm{H}$ NMR spectroscopy, was readily varied by varying the nucleophile composition. The molecular weights of all the precursors were found to be very high; $\mathrm{M}_{\mathrm{w}}$ lies in the range $220 \mathrm{kDa}$ to $260 \mathrm{kDa}$, as determined by GPC using polystyrene standards.

\subsection{Solution photophysical properties}

The solution absorption and fluorescence spectra of segmented MEHPPV- $x$ are shown in figure 1. It is apparent that there is a continuous bathochromic shift in the absorption and emission maxima with increase in the extent of conjugation. Also, as the conjugation length increases, the emission from different individual conjugated segments disappears and a continuously narrowing emission peak is observed (for MEHPPV-69 and above).

The quantum yield of fluorescence $\left(\phi_{\mathrm{f}}\right)$ (relative to standard quinine sulfate) also decreased monotonically with increase in the conjugation length (figure 2 ). This is in contrast to that observed in the case of pure oligomers OPV- $n$, where the $\phi_{\mathrm{f}}$ value was observed to attain a maximum for OPV-3 and then decreases before it levels off at around 0.4 after OPV-6 [14]. This difference is reflective of the presence of a statistical distribution of conjugation lengths in the case of polymers, and is further compounded by the occurrence of intra-chain energy transfer, as we shall see later. 


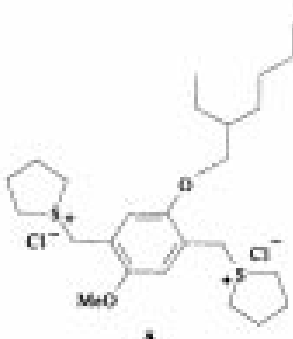

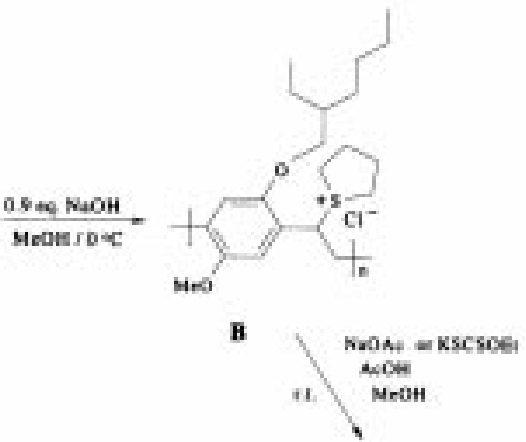
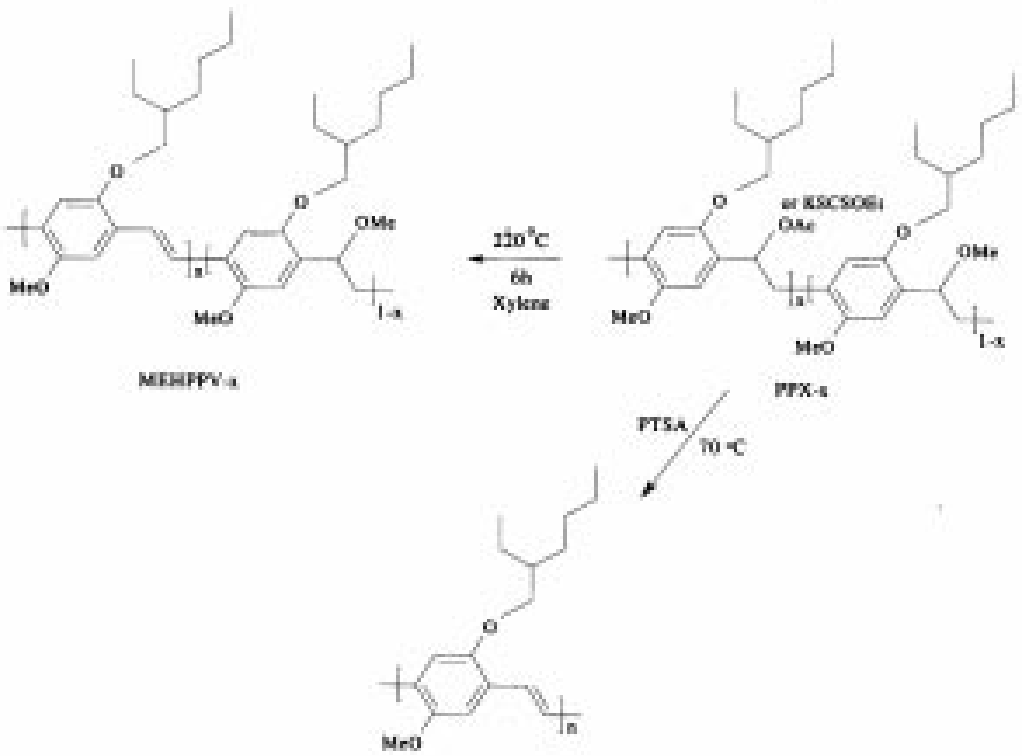

wrX-s

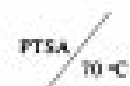

Scheme 1. Synthesis of MEHPPV- $x$ and subsequent conversion to MEHPPV-100 via MAP route.

In our approach to control conjugation length, we rely on the statistically random placement of acetate (or xanthate) and methoxy groups in the precursor during the competitive nucleophilic substitution of the Wessling polyelectrolyte, and further on the selective elimination of the acetate groups. This would lead to the generation of truncated MEHPPV oligomers of varying conjugation lengths even within a given polymer chain, the average value of which increases with increasing extents of elimination. In an effort to ascertain the nature of this distribution of conjugation lengths, we reconstructed the absorption spectra of the polymers using those of the various OPV- $n$ oligomers, assuming a statistically random elimination process [12]. The individual probabilities of finding the oligomer segments of various conjugation lengths, in each of the MEHPPV- $x$ samples, were thus calculated and the absorption spectra of the OPV- $n$ oligomers [15] were factored (to reflect this expected oligomer concentrations) and summed up to generate the reconstructed absorp- 

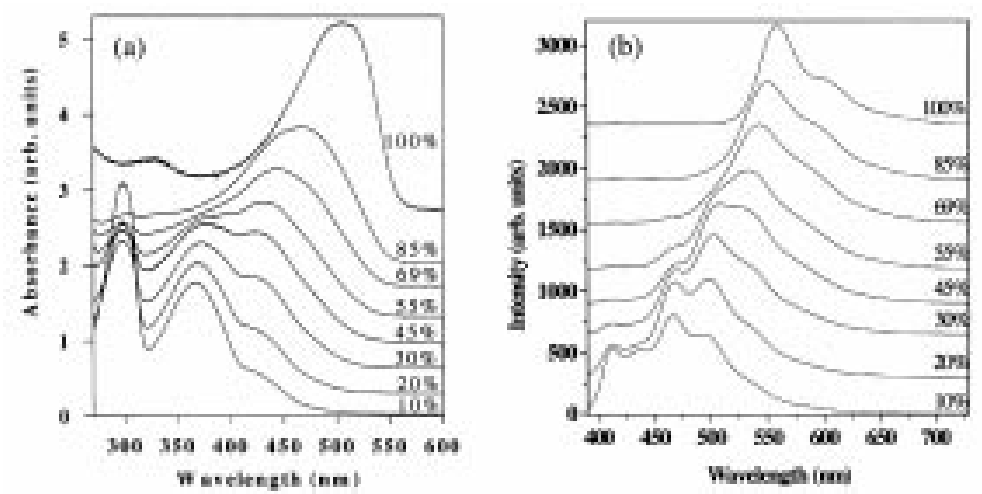

Figure 1. Solution UV-visible absorption (a) and emission (b) spectra of copolymers MEHPPV- $x$. The spectra are normalized in intensity and have been Y-offset for clarity.

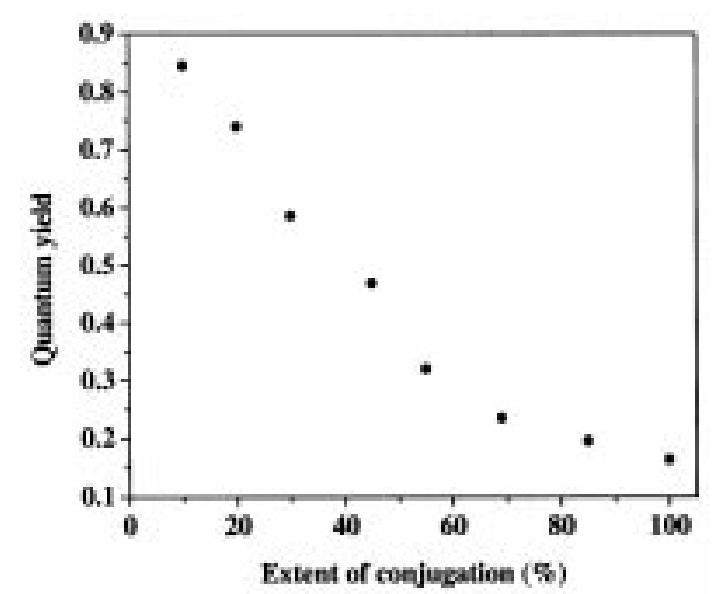

Figure 2. Variation of fluorescence quantum yield, $\phi_{\mathrm{f}}$ (ca. $10^{-6} \mathrm{M} \mathrm{CH}_{2} \mathrm{Cl}_{2}$ solutions, $\lambda_{\text {excitation }}=370 \mathrm{~nm}$ ) of copolymers MEHPPV- $x$ with $x$.

tion spectra. A few such reconstructed spectra alongside the measured ones are shown in figure 3a. The reconstructed spectra matched very well with the observed ones in as much as the relative populations of the various oligomers are concerned (as seen from the overall similarity in their shapes), although the measured spectra, in many cases, are significantly red-shifted $(\sim 12-15 \mathrm{~nm})$. This is possibly due to an intrinsic difference between the $\lambda_{\max }$ values for the actual MEHPPV oligomers and those for OPV- $n$ oligomers, which is also reflected in the ca. $26 \mathrm{~nm}$ difference in $\lambda_{\max }$ of poly(2,5-dipropyloxyphenylene vinylene) when compared to MEHPPV-100. Despite these limitations, the overall one-to-one correspondence in shape between the reconstructed and the observed absorption spectra amply supports the hypothesis of a statistically random evolution of conjugation lengths in the MEHPPV- $x$ samples.

In an effort to understand energy transfer in solutions of conjugated polymers, the fluorescence spectra of MEHPPV- $x$ were also reconstructed using a similar statistical weigh- 

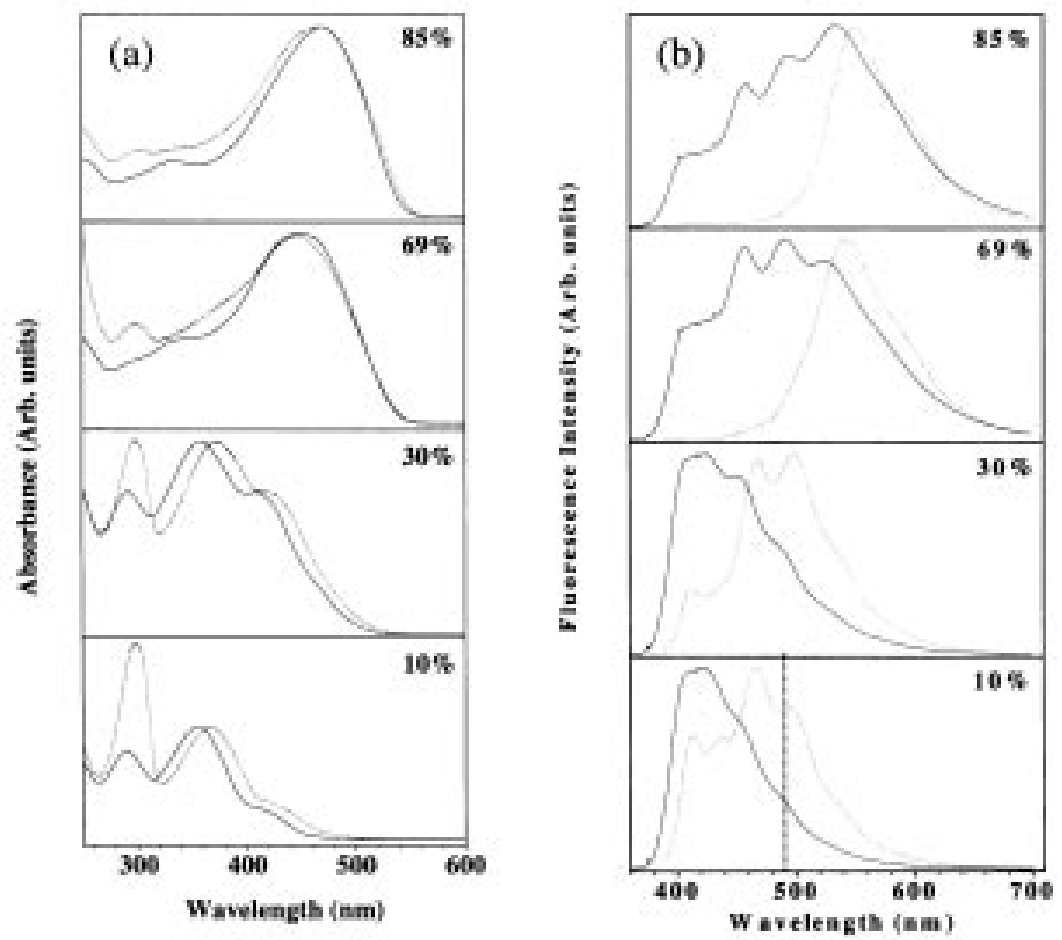

Figure 3. Overlay of recorded (broken lines) and reconstructed (continuous lines) UV-visible absorption (a) and emission (b) spectra of MEHPPV- $x$.

ing and summing up of the various OPV- $n$ fluorescence spectra. Unlike in the case of the absorption spectra, the reconstructed fluorescence spectra differ considerably from the observed ones in all cases (see figure 3b). The spectra of MEHPPV- $x$ samples often lack the well-resolved fine structure (especially in highly eliminated samples) that reflects separate emission from the individual chromophores of varying conjugation lengths, and also majority of the emission occurs at considerably lower energy when compared to the reconstructed spectra. Both these aspects reflect the occurrence of energy transfer. As these spectra were recorded under very dilute conditions, the energy transfer may be assumed to occur primarily via an intra-chain pathway [16].

\subsection{Solvent-induced conformational transition}

It has been known for sometime that the morphological effects arising from processing from different solvents affect the photophysical and electroluminescence properties of soluble PPV derivatives significantly [17]. From steady state and time-resolved fluorescence spectroscopic studies of MEHPPV as a function of the nature of the solvent, Collison et al [18] have shown that there exist only two species in solution for MEHPPV - the completely free chains and aggregates. Therefore, the high sensitivity of the solution fluorescence 
spectra of MEHPPV to the chain conformation could serve as an excellent tool to examine their conformational changes in dilute solutions. Segmented MEHPPV- $x$, with varying extents of conjugation may be perceived as a collection of fluorophores of varying conjugation length (energies) that are confined to a relatively small volume as a result of being tied together in a single polymer chain. As we had seen earlier, rapid intra-chain energy migration happens even in very dilute solution, resulting in a significant red-shifted emission accompanied by a lowering of emission yields. Thus, apart from aggregation effects seen by earlier workers in MEHPPV due to solvent variation, in segmented MEHPPV-x, one can examine the variation in extent of energy transfer due to conformational changes induced by solvent variation. It is also important to recognize that the stiffness of the polymer chain is also likely to vary significantly as the extent of conjugation is increased - going from a relatively flexible chain at lower extents of conjugation to a stiff chain at higher extents of conjugation. To probe these effects, the fluorescence spectra of segmented MEHPPV- $x$ were examined in $\mathrm{CH}_{2} \mathrm{Cl}_{2}-\mathrm{MeOH}$ solvent-nonsolvent pair, as a function of methanol composition [19].

The fluorescence spectra of MEHPPV-100 recorded at a concentration of $3 \times 10^{-6} \mathrm{M}$, as a function of varying volume fractions of methanol are shown in figure 4a. Two distinct features are apparent from the figure: (i) there is significant red-shift in the emission maximum at high methanol content and (ii) a drastic decrease in the overall emission yield with increasing methanol content. Upon careful examination it is clear that the main peak at ca. $560 \mathrm{~nm}$ depletes rapidly in intensity, while the shoulder to the red becomes the dominant peak beyond a certain concentration $(>30 \%)$ of methanol.

In order to follow this variation more quantitatively, the variation in the total area under the emission envelop (emission yield) as well as the emission maximum $\left(\lambda_{\text {max,emi }}\right)$ are plotted as a function of methanol composition (figure 4b). In both cases, a sudden change occurring over a small composition variation is seen, which is suggestive of a co-operative effect reminiscent of the conformational transitions observed in biopolymers. This kind of sudden change cannot be ascribed to simple solvatochromic effects but is clearly suggestive of a drastic change in the conformation of the polymer chains, which in turn, affects parameters such as aggregation, energy transfer, interchain exciton formation, etc. The
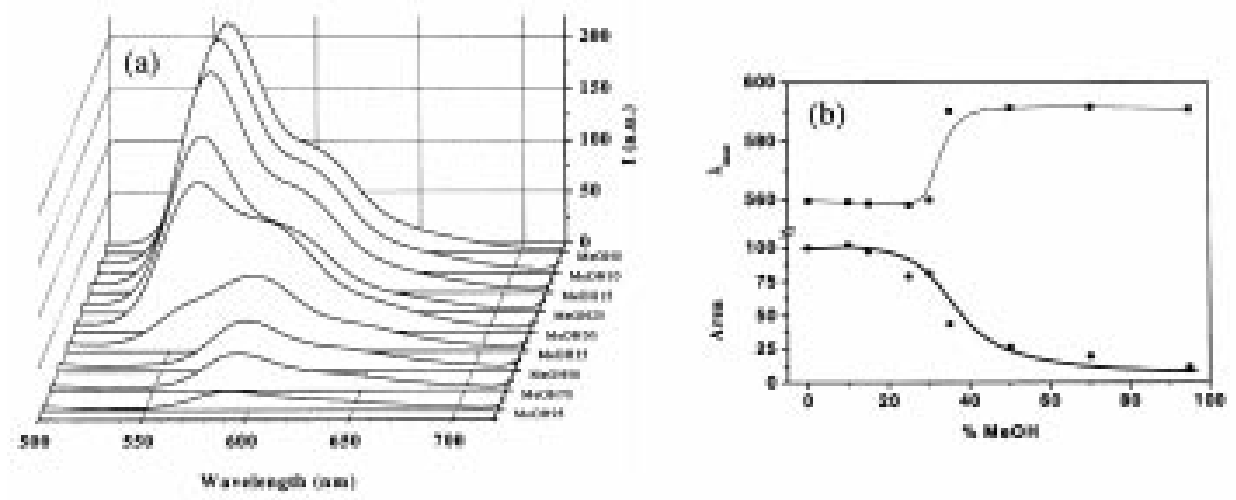

Figure 4. (a) Fluorescence spectra of MEHPPV-100 at various volume fractions of methanol in dichloromethane. (b) Change in the $\lambda_{\max }$ and emission yield upon addition of methanol. 
inflexion point of the S-shaped curves is taken as the 'chain collapse point'. Since these measurements are carried out at very dilute solution $\left(\sim 10^{-6} \mathrm{M}\right)$, these variations can be ascribed to an isolated single-chain conformational transition.

We carried out similar studies for four different segmented MEHPPV- $x$ samples with varying extents of elimination, where $x=5,10,30$, and 55. In case of MEHPPV-5, there are only two peaks at $415 \mathrm{~nm}$ and $445 \mathrm{~nm}$, to start with, along with a weak tail extending to longer wavelengths (figure 5). With increasing amounts of methanol, a shoulder at a higher wavelength begins to evolve and eventually becomes the dominant peak with an emission maximum at ca. $470 \mathrm{~nm}$.

In the case of MEHPPV-10, the fluorescence spectrum even in pure $\mathrm{CH}_{2} \mathrm{Cl}_{2}$ exhibits distinct peaks due to both stilbene-type (at $415 \mathrm{~nm}$ ) and distilbene-type units $(460 \mathrm{~nm})$ with a tailing indicative of the presence of other higher homologues. As the volume fraction of methanol is increased, as expected, one sees a sudden decrease in the emission yield going through a similar S-shaped curve, as in the other MEHPPV- $x$ samples; the inflexion point in this case occurs at a slightly lower value than MEHPPV-5. An additional intriguing feature in this case is that, there is a very clear increase of emission yield at the early stages, after small quantities of methanol were added, prior to the sudden decrease.

On careful examination of the expanded region of the spectral evolution (figure 6a) a rather unprecedented feature becomes apparent. Initially as the intensity of $415 \mathrm{~nm}$ emission band decreases, the intensity of the peak at $460 \mathrm{~nm}$ increases. Beyond a certain concentration of methanol, as expected, the intensity of all the emission bands decreases drastically, as seen in figure 5b. In order to explain this behavior, one needs to look at the variation of $\phi_{\mathrm{f}}$ of well-defined oligomers. The fluorescence quantum yields of oligomeric dipropyloxy-PPVs (OPV- $n$ ) were reported to exhibit a rather unusual variation as a function of oligomer length (figure 6b); the smallest oligomer, stilbene (OPV-1), has a fluorescence quantum yield of ca. 0.45 , which goes up to about 0.8 for distilbene (OPV-2) and further to ca. 0.81 for OPV-3, before falling drastically for even higher oligomers [14]. It remains essentially constant for oligomers with $n \geq 6$.
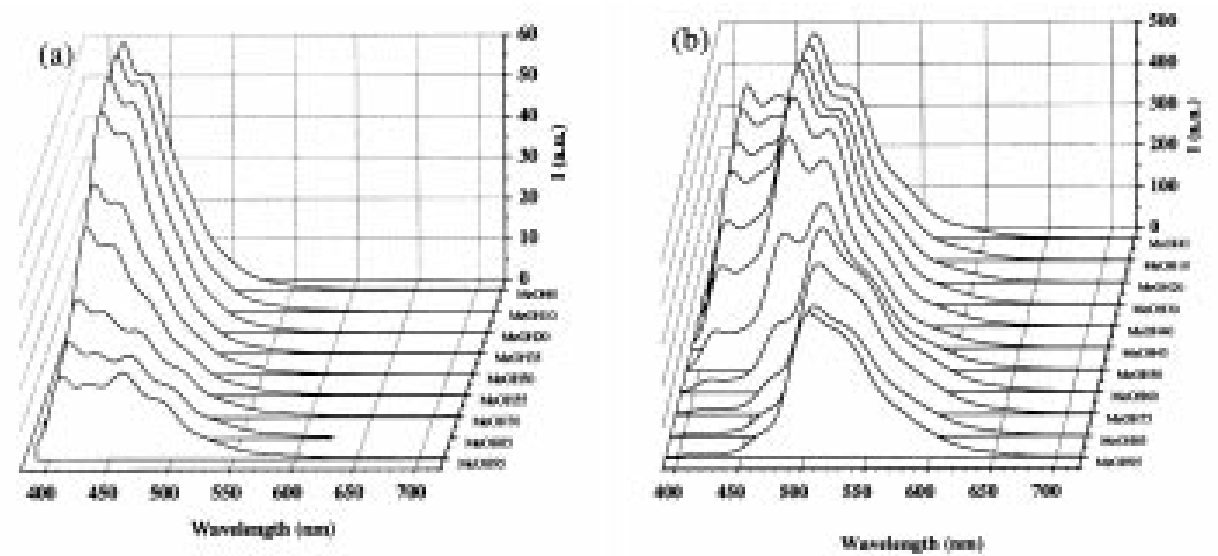

Figure 5. Fluorescence spectra of MEHPPV-05 (a) and MEHPPV-10 (b) at different $\%$ volume fractions of $\mathrm{MeOH}$. 
Given this rather intriguing variation of quantum yields of OPVs, the origin for the initial enhancement of emission yield in MEHPPV-10 is readily explained. Energy transfer from a stilbene-type chromophore to a distilbene/tristilbene-type ones results in the generation of an excited state with intrinsically higher quantum yield for emission. Hence, while there is a lowering of the intensity of the peak due to the stilbene-type unit, there is an enhancement of the peak due to distilbene-type (and probably tristilbene-type too). However, at much higher concentrations of methanol, the emission yield decreases due to energy transfer to higher oligomers $(n>4)$ and/or due to the formation of intra-chain inter-chromophore excitons.

Similar studies, as a function of solvent composition, were carried out for two other MEHPPV- $x$ samples, namely MEHPPV-57 and MEHPPV-30 and a plot of the variation of the so-called 'chain-collapse point' as a function of extent of conjugation $x$, in MEHPPV- $x$, is shown in figure 7. It is clear from this plot that as one increases the extent of conjugation, the amount of non-solvent required to effect chain collapse decreases.

Earlier workers who have studied fully conjugated MEHPPV have ascribed the sudden drop in fluorescence yield to the dimensional change of the polymer chain leading to enhanced formation of intra-chain inter-chromophore excitons (aggregates) which have considerably lower emission quantum yields [16]. In segmented MEHPPV- $x$ samples, however, apart from the enhanced probability of aggregate formation, energy transfer to higher oligomers with lower emission efficiency is also important.

Since the starting molecular weights of all the polymers are roughly the same, (especially since all of the precursor polymers with varying value of $x$ are made from a single polymeric intermediate, namely the Wessling precursor) the initial hydrodynamic volume of the various MEHPPV- $x$ samples may be taken as the same. Hence, if the variation in the hydrodynamic volume for different segmented MEHPPV- $x$ samples were to follow identical paths, the effective chromophore concentrations being higher when $x$ is larger, one sees an apparent shift in the chain-collapse point. In other words, if we were to assume that, in as far as the fluorescence measurements are concerned, the collapse is visualized as soon as the average inter-chromophore distance reaches a certain threshold value $r$, then this threshold is reached at an earlier stage (larger hydrodynamic volume) for highly conjugated samples (larger values of $x$ ) than for less conjugated ones.
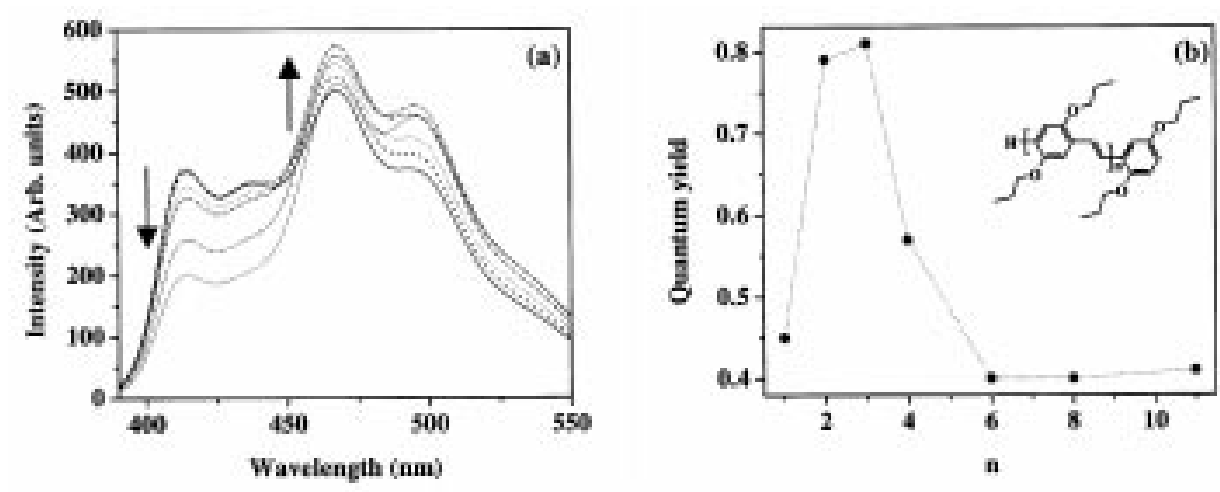

Figure 6. (a) Expanded region of the variation of fluorescence spectra of MEHPPV-10 as a function of methanol. (b) Variation of fluorescence quantum yield of OPVs as a function of oligomer length $n$ (adapted from [16]). 


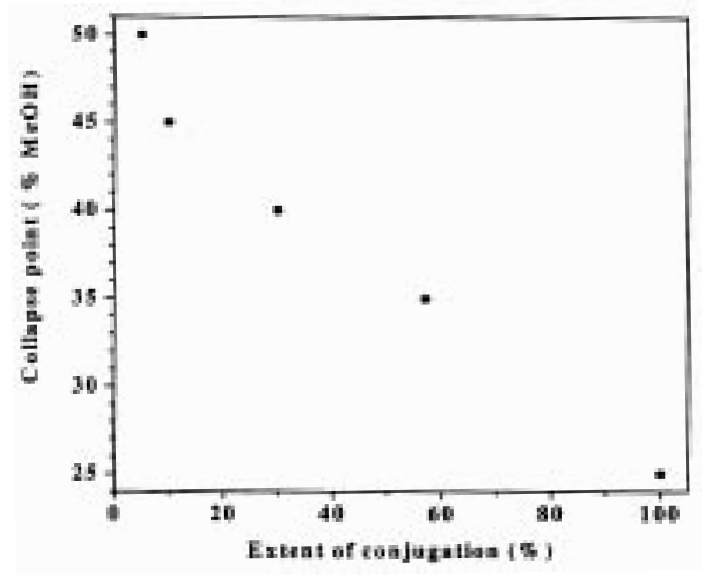

Figure 7. Effect of conjugation on the chain collapse point for MEHPPV-x.

In essence, our argument therefore gives greater weightage to the variation in the average inter-chromophore distance, assuming that intrinsic variations in the hydrodynamic volumes of segmented MEHPPV- $x$, as a function of $x$ is less important. Needless to add that independent verification of this is possible only if simultaneous measurements of sizes are also made.

\section{Conclusions}

Segmented MEHPPV- $x$ samples present unique opportunities to probe and understand conformational transitions in polymer molecules using simple steady-state fluorescence measurements. As the composition of the solvent-medium becomes richer with respect to the non-solvent, a sudden drop in the emission yield is noticed. This drop is ascribed to a sudden increase in the number of poorly emissive inter-chromophore excitonic species formed within a single polymer chain. Increasing the extent of conjugation in MEHPPV$x$ increases the chromophore density within the hydrodynamic volume of the polymer molecule, and hence the apparent chain collapse point happens at lower non-solvent composition. As these measurements are carried out at very dilute polymer concentrations, they are a direct consequence of single chain conformational transitions. An alternate way to affect such a conformational collapse is by variation of temperature, which has the added advantage of being able to readily examine the reversibility of such transitions. Studies are currently underway to examine such temperature-induced transitions and will be reported shortly.

\section{Acknowledgements}

We would like to thank CSIR, New Delhi, for their generous support through an extramural grant (No: 01(1698)/01/EMR-II). 


\section{References}

[1] J H Burroughes, D D C Bradley, A R Brown, H N Marks, K Mackay, R H Friend, P L Burn and A B Holmes, Nature (London) 347, 539 (1990)

[2] R H Friend, R W Gymer, A B Holmes, J H Burroughes, R N Marks, C Taliani, D D C Bradley, D A dos Santos, J L Bredas, M Logdlund and W R Salaneck, Nature (London) 397, 121 (1999)

[3] J J M Halls, C A Walsh, N C Greenham, A B Holmes, E A Marsegila, R H Friend and S C Moratti, Nature (London) 376, 498 (1995)

[4] N Tessler, G J Denton and R H Friend, Nature (London) 382, 695 (1995)

[5] N S Sariciftci, L Smilowitz and A J Heeger, Science 258, 1474 (1992)

[6] A R Brown, N C Greenham, J H Burroughes, D D C Bradley, R H Friend, P L Burn, A Kraft and A B Holmes, Chem. Phys. Lett. 200, 46 (1992)

[7] C Zhang, D Braun and A J Heeger, J. Appl. Phys. 73, 5177 (1993)

[8] P L Burn, A B Holmes, A Kraft, D D C Bradley, A R Brown, R H Friend and R W Gymer, Nature (London) 356, 47 (1992)

[9] P L Burn, A B Holmes, A Kraft, D D C Bradley, A R Brown and R H Friend, J. Chem. Soc., Chem. Commun. 32 (1992)

[10] D Braun, E G J Staring, R C J E Demandt, G L J Rikken, Y A R R Kessener and A H J Venhuizen, Synth. Met. 66, 75 (1994)

[11] R Gowri, D Mandal, B Shivkumar and S Ramakrishnan, Macromolecules 31, 1819 (1998)

[12] G Padmanaban and S Ramakrishnan, J. Am. Chem. Soc. 122, 2244 (2000)

[13] G Padmanaban and S Ramakrishnan, Synth. Met. 119, 533 (2001)

[14] U Stalmach, Dissertation Thesis (University of Mainz, 1996)

[15] H Meier, U Stalmach and H Kolshorn, Acta Polym. 48, 379 (1997)

[16] M Yan, L J Rothberg, E W Kwock and T M Miller, Phys. Rev. Lett. 75, 1992 (1995)

[17] T-Q Nguyen, I Martini, J Liu and B J Schwartz, J. Phys. Chem. B104, 237 (2000)

[18] C J Collison, L J Rothberg, V Treemaneekarn and Y Li, Macromolecules 34, 2346 (2001)

[19] G Padmanaban and S Ramakrishnan, (under preparation) 